\title{
Interlayer Mass Transport and Quantum Confinement of Electronic States
}

\author{
M. Giesen,* G. Schulze Icking-Konert, and H. Ibach \\ Institut für Grenzflächenforschung und Vakuumphysik, Forschungszentrum Jülich, D-52425 Jülich, Germany
} (Received 17 November 1998)

A numerical analysis of the ripening of multilayer islands on $\mathrm{Cu}(111)$ shows that the EhrlichSchwoebel barrier for interlayer mass transport is independent of the terrace width $w$ as long as $w>w_{c}=14 \pm 2 \AA$, but vanishes for $w<w_{c}$. The critical width $w_{c}$ corresponds exactly to the terrace width below which the surface state is pushed above the Fermi level due to quantum confinement. The Ehrlich-Schwoebel barrier is therefore correlated with the occupation of surface states. [S0031-9007(99)08912-7]

PACS numbers: 68.35.Fx, 73.20.Dx

The growth of atomically flat epitaxial films requires a facile mass transport across the step edges which separate terraces of different height. Diffusion across a step edge is frequently hindered by an activation barrier for interlayer mass transport (Ehrlich-Schwoebel barrier [1,2]). The existence of a significant EhrlichSchwoebel barrier (ES barrier) is well documented for the (111) surfaces of $\mathrm{Pt}, \mathrm{Rh}, \mathrm{Ag}$, and $\mathrm{Cu}$ [3-7]. In these and other experiments, merely an average ES barrier is typically determined. Total energy calculations based on the embedded atom model (EAM) [8-10] as well as recent $a b$ initio calculations in the local density approximation [11] have shown that activation energies for mass transport across a step depend on the type of steps and involve an exchange of the adatom with a step atom. For the experimentally rather extensively studied Pt(111) surface Feibelman [11] has shown that the activation energy is practically zero $(0.02 \mathrm{eV})$ for crossing the $A$ step [displaying a (100) facet], but large $(0.35 \mathrm{eV})$ for the $B$ step [(111) facet]. This result is at variance with the large effective ES barrier observed in earlier experiments [3]. However, according to a recent study [12] the large ES barrier in earlier work was caused by preferential adsorption of $\mathrm{CO}$ on $A$ steps from the residual gas. On clean $\mathrm{Cu}(111)$ surfaces on the other hand, a significant ES barrier was found experimentally also for the $A$ type step [13]. EAM calculations have further revealed significant differences in the ES barrier on a straight step and at kink sites $[9,10]$. Whether the calculated differences are always significant for the interlayer mass transport depends on the preexponential factors which are presently not known and may (partly) compensate different activation barriers (see, e.g., [14]).

Faced with the unpleasantly complex situation, both on the experimental and theoretical side, a better understanding as to why and when larger ES barriers exist, would be rather welcome. An attempt in that direction was made several years ago by Memmel and Bertel [15] who conjectures that the existence of an ES barrier on (111) oriented fcc surfaces is correlated with the nearly free electron surface state [16]. It was argued that the boundary condition for the surface state wave function at the steps leads to a reduction of the binding energy at steps and hence to an ES barrier. While the concept is appealing, convincing evidence for a correlation between the existence of surface states and the ES barrier is lacking. A search for a possible correlation could proceed basically on two routes. One would be to study binding energies and activation energies in ab initio calculations such as [11] in connection with the electronic structure of surfaces. A second route would be to modify experimentally the charge density contributed by the surface state and search for changes in the ES barrier. Steps on (111) surfaces act as a nearly infinite potential boundary for the surface state [17] which opens up an elegant possibility to modify the charge density in the surface state without changing other parameters: By confining the surface state wave function to a narrow terrace between two steps the ground state energy of the surface state is pushed above the Fermi level and hence the occupation of the surface state changes from the maximum value on large terraces to zero. Hence, a determination of the magnitude of the ES barrier as a function of terrace width (TW) can establish whether a correlation between the charge density in the surface state and the ES barrier does exist or not. In this paper we report on such an experiment: We have measured the mass transport across steps in multilayer islands as a function of the width of the respective upper terrace adjacent to the step. The ES barrier (activation energy and prefactor) is determined using a numerical analysis of the island decay. We find that, independent of temperature, the ES barrier vanishes rather abruptly for terrace widths smaller than $14 \pm 2 \AA$ which corresponds to the width where the confinement pushes the surface state band above the Fermi level.

Island decay on $\mathrm{Cu}$ (111) was analyzed using continuous STM recording in a vacuum chamber (base pressure below $5 \times 10^{-11}$ mbar). The STM setup and the crystal preparation was described in [13]. The observed surface density of pinning sites was $10^{-7}$ per surface atom and the surface remained clean after $10 \mathrm{~h}$ of observation. Before deposition, the $\mathrm{Cu}$ evaporator was carefully outgassed for about $15 \mathrm{~min}$. The pressure never exceeded $2 \times$ $10^{-10}$ mbar during $\mathrm{Cu}$ deposition. In each experiment 
30-50 monolayers (ML) of $\mathrm{Cu}$ were deposited at a rate of $0.5 \mathrm{ML} / \mathrm{s}$ while the surface was held at $300 \mathrm{~K}$. Under these conditions, mounds of 2-4 ML height are formed. Then, the sample was radiatively heated to the desired temperature and mounted on the microscope. Thermal equilibrium of the sample as well as a stable tunneling condition was achieved $5 \mathrm{~min}$ after $\mathrm{Cu}$ deposition. For $T<320 \mathrm{~K}$, the decay of multilayer mounds was observed in up to 1200 STM images, covering a total time span of about $10 \mathrm{~h}$. Because of the larger decay rates, the number of STM images was smaller for higher temperatures. For the highest temperature $(382 \mathrm{~K})$, mound decay was observable in 5-10 STM images for a total time span of about $5 \mathrm{~min}$. At each temperature, we have analyzed the decay of 2-4 different mounds. The islands retain their equilibrium shape during the decay due to the rapid mass transport along the perimeter.

As an example, Fig. 1(a) shows the measured areas of the top (circles) and the 2nd (squares) layer island in a double layer mound at $T=303 \mathrm{~K}$ vs time. The inset of Fig. 1(a) displays the corresponding STM image (scan area $680 \times 680 \AA^{2}$ ) at $t=6600 \mathrm{~s}$. Initially, the top layer island is significantly smaller than the 2 nd layer island. In that stage, the decay of the top layer island is slow compared to the 2nd layer island due to the ES barrier which hinders the interlayer mass transport. At around $t=6600 \mathrm{~s}$, the difference in the island areas of the 2nd and the top layer island has become smaller and the TW has approached a critical value of about $14 \AA$. Then, the decay
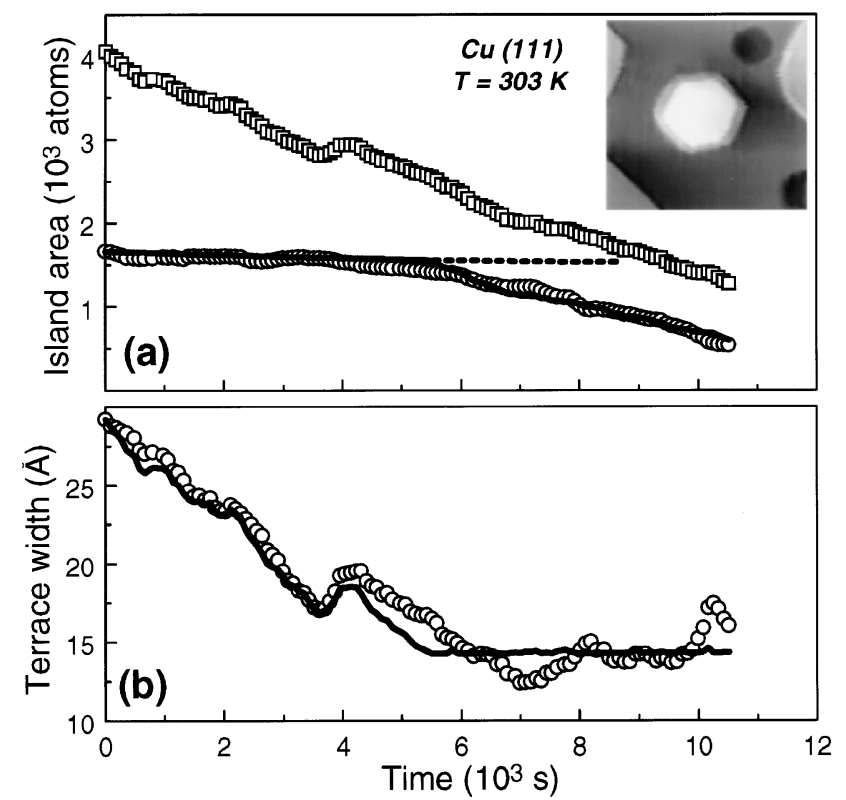

FIG. 1. (a) Areas of the top and bottom layer of a two-layer island (see inset) vs time are denoted as circles and squares, respectively. The "hiccup" in the area of the bottom layer island at $t=4000 \mathrm{~s}$ is due to the final decay of a nearby situated island. (b) Terrace width $w$ between the top and bottom layer island (see text for discussion of the solid and dashed lines). rate of the top layer island accelerates and the TW remains approximately constant save for some wiggles [Fig.1(b)]. The magnitude and the time structure of these wiggles are different in each decay curve studied. The wiggles are therefore presumably caused by the random walk of the top layer island and the changing environment of the lower island. The acceleration of the decay of top layer islands as well as the constant TW in the late stages of the decay has been shown previously [18]. It was speculated that the increased decay rate of the top layer island may be caused by the formation of microscopic contacts between the island edges due to thermal edge fluctuations and the stochastic motion of the islands. These ledge contacts with a possible formation of microfacets could provide rapid channels for interlayer mass transport, bypassing the ES barrier. Since the edge fluctuations and the random motion of the island increase exponentially with temperature, the model predicts the mean TW during the final decay to increase with temperature. The result of this work is that the TW is independent of temperature: Figure 2 shows an Arrhenius plot of the mean TW during the late stage of mound decay. Each data point corresponds to a complete decay curve for an island stack such as in Fig. 1. The error bars are the standard deviation from the mean. Since there is no measurable temperature dependence of $w$ in the late stage of decay, we can disregard the hypothesis that island edge fluctuations and island wandering are involved in the process leading to rapid mound decay. From the experimental results we obtain a mean TW of $14.2 \pm 2.1 \AA$.

In order to elucidate the relation between the magnitude of the ES barrier and the terrace width $w$, we have analyzed the decay curves of multilayer mounds as a function of temperature in detail. In a first step, we considered the decay of small top layer islands via the normal, ES-barrier controlled, interlayer mass transport. It is straightforward to show that the decay is described by the rate equation $[13,19]$

$$
\frac{d \hat{\mathrm{R}}_{t}}{d t}=\frac{\nu_{0} e^{-E_{\mathrm{diff}} / k_{B} T}}{\hat{\mathrm{R}}_{t}} \frac{\rho_{\mathrm{eq}}\left(\hat{\mathrm{R}}_{t}\right)-\rho_{\mathrm{eq}}\left(\hat{\mathrm{R}}_{b}\right)}{\ln \left(\hat{\mathrm{R}}_{b} / \hat{\mathrm{R}}_{t}\right)+(1-s) / s \hat{\mathrm{R}}_{b}} .
$$

Here, $\hat{\mathbf{R}}_{t}$ and $\hat{\mathbf{R}}_{b}$ are the distances of the "straight" sections of the hexagonal top and the bottom layer islands to the center in units of the square root of an atom area and

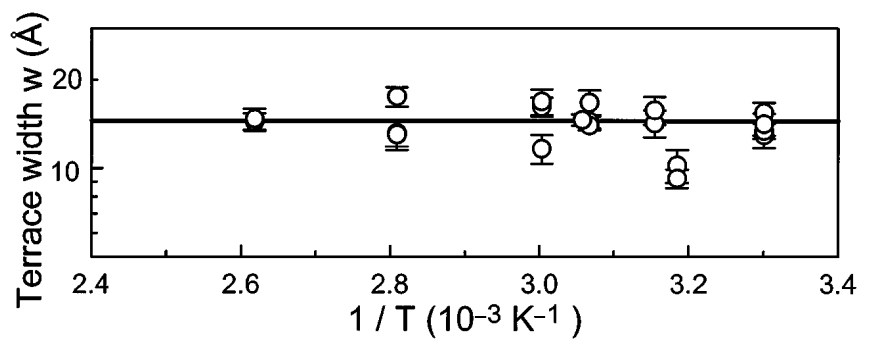

FIG. 2. Temperature dependence of the terrace width $w$ of multilayer islands in the late stages of decay. 
$\nu=\nu_{0} e^{-E_{\mathrm{diff}} / k_{B} T}$ is the hop frequency of atoms on the terraces with $E_{\text {diff }}$ the activation energy for diffusion. The "sticking coefficient" $s$ is the ratio of the step and terrace hop frequencies and involves the activation energy $E_{\mathrm{ES}}$ and the dimensionless number $s_{0}$ :

$$
s(T)=s_{0} \exp \left(-E_{\mathrm{ES}} / k_{B} T\right) .
$$

The equilibrium concentrations of adatoms at the island edges $\rho_{\text {eq }}(\hat{R})$ are given by the energy $E_{a}$ to create an adatom from a kink site and the free energy $\tilde{\gamma}$ necessary to elongate the island perimeter by one atom.

$$
\rho_{\mathrm{eq}}(\hat{\mathrm{R}})=e^{-E_{a} / k_{B} T} e^{\tilde{\gamma} / \hat{\mathrm{R}} k_{B} T} .
$$

The product of the hop frequency $\nu$ and the equilibrium concentration for a straight step $\rho_{\text {eq }}(\hat{\mathrm{R}}=\infty)$ and $\tilde{\gamma}$ were determined previously in independent experiments which involved the decay of islands in vacancy islands as well as the decay of entire ensembles of islands and a numerical analysis of the diffusion equation $[13,19]$. The activation energy $E_{a}+E_{\text {diff }}$ was found to be $0.76 \pm 0.04 \mathrm{eV}$ [13], the preexponential factor was $\nu_{0}=1 \times 10^{12 \pm 0.65} \mathrm{~s}^{-1}$ $[13,19]$, and the line tension $\tilde{\gamma}=0.53 \mathrm{eV}$. We note that this line tension differs from the line tension $(0.22 \mathrm{eV})$ [20] which serves as a restoring force in equilibrium fluctuations. The deviation, far outside the experimental error, poses an interesting problem which is, however, beyond the scope of this paper.

We have analyzed the decay of about 40 multilayer $\mathrm{Cu}$ islands on $\mathrm{Cu}(111)$ between $T=298$ and $382 \mathrm{~K}$ using the independently determined values [13,19] for $E_{a}+E_{\text {diff }}$, $\nu_{0}$, and $\tilde{\gamma}$. Experimental data for the decay of an island at a particular temperature were fitted to Eq. (1) by varying the sticking coefficient $s$ (details will be published in a forthcoming paper) [21]. From an Arrhenius plot of all data for $s(T)$ we obtain

$$
\begin{aligned}
E_{\mathrm{ES}} & =0.224 \pm 0.009 \mathrm{eV}, \\
s_{0} & =3.5 \times 10^{ \pm 0.35} .
\end{aligned}
$$

The error is the statistical error. Possible errors in $E_{a}+E_{\mathrm{diff}}, \nu_{0}$, and $\tilde{\gamma}$ are not taken into account. We note that the numbers represent a weighted average over the two types of steps bearing a temperature dependent concentration of kink sites. The result is consistent with the sticking coefficient found for the $A$ step [13]. The prefactor $s_{0}$ found here is significantly lower than for $\operatorname{Ag}(111)$ [22].

Using the above values for $s_{0}$ and $E_{\mathrm{ES}}$, the dashed line in Fig. 1(a) is calculated for the decay of the top layer when the effective radius $\hat{\mathrm{R}}_{b}$ of the 2 nd layer island is taken from the experiment. Otherwise, no parameter is fitted to the individual data set. The dashed line initially matches the experimental decay curve as it should, however, it deviates when the TW becomes smaller than about $14 \AA$. The decay curve in Fig. 1(a) and the decay curves for all other investigated island (total of 20 islands) are well described if one assumes that the sticking coefficient $s$ changes abruptly from $2.6 \times 10^{-3}$ (at $T=$ $303 \mathrm{~K}$ ) to 1 when the mean TW between the upper and lower island is below $w_{c}=14 \AA$ (solid line). The change in the decay rate in the experimental data is somewhat smoother than the change of the slope in the calculated curve. The data are, nevertheless, consistent with the assumption of an abrupt transition since the wandering of the top island temporarily leads to TWs smaller than $14 \AA$ even when the mean TW is still larger. The numerical calculation for the particular top island shown in Fig. 1(a) would also match the experiment if a more moderate reduction of the ES barrier had been assumed. However, the rapid decay occasionally observed when the top layer islands accidentally comes very close to the boundary of the lower island [18] requires that the ES barrier vanishes more or less completely. We point out that the data cannot be described by assuming an enlarged diffusion constant on small terraces since the activation energy for diffusion is already small (about $0.05 \mathrm{eV}[23,24]$ ). In summary our data analysis shows (i) a constant ES barrier for TW larger than $w_{c}$, (ii) a reduction of the barrier to zero or to a small value as soon as the TW is below $w_{c}=14 \pm 2 \AA$.

The critical width $w_{c}$ corresponds to a terrace of six atom rows. The size of the critical TW as such, as well as the fact that $w_{c}$ is independent of temperature excludes interpretation schemes involving a barrier reduction by a temporary formation of microfacets. The rather abrupt transition in $s$ for $w=w_{c}$ also excludes models which involve the interaction of the local strain fields around steps, as these strain fields vary smoothly with the distance. The only quantity which does change abruptly at a TW of $14 \AA$ is the density of occupied electronic states. On the $\mathrm{Cu}(111)$ surface, the image potential surface state obeys a parabolic dispersion $E(k)-E_{F}=\left(8.25 \AA^{2} \mathrm{eV}\right) k^{2}-0.389 \mathrm{eV}$ [16]. Li et al. [17] have shown that the wave function of the surface state has a node at steps. Because of the node the minimum wave vector $k_{\min }$ for terraces between the steps is $k_{\min }=\pi / \mathrm{TW}$. The ground state energy of the surface state is therefore pushed upwards for small TWs, eventually above the Fermi level.

In Fig. 3 the number of electrons per atom in the surface state is plotted as a function of the TW assuming that the other dimension of the terrace is infinitely long, i.e., we assume a one-dimensional density of states. This is a reasonable representation of the experimental situation [see inset of Fig. 1(a)]. The number of electrons in surface states is zero below a TW of $14.5 \AA$ and reaches $50 \%$ of its maximum value at $16.1 \AA$. Hence one has an abrupt transition in the occupation of the surface state exactly at the TW where we find the ES barrier to vanish. Dismissing the possibility of that being a sheer coincidence of numbers, we conclude that the occupation of surface states affects the ES barrier on the $\mathrm{Cu}(111)$ surface. We propose this conclusion to be pertinent 


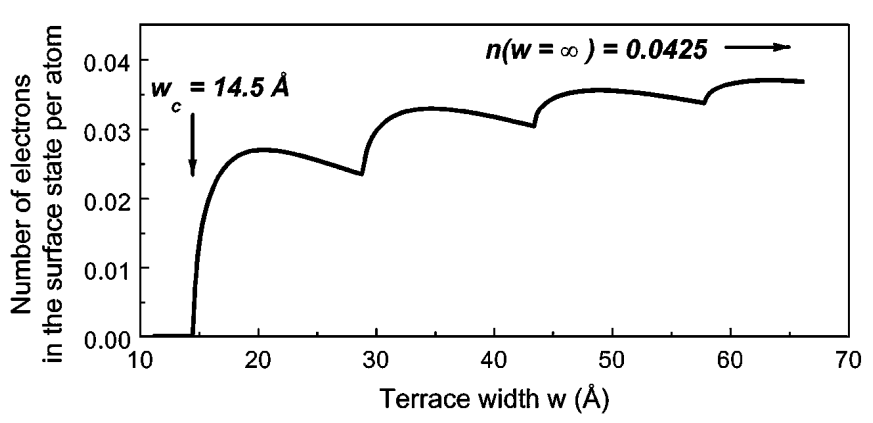

FIG. 3. Number of surface state electrons per atom on the terrace between two island edges. No surface states are occupied below $w_{c}=14.5 \AA$ as the energy of the ground state is above the Fermi level because of the quantum confinement. The cusps at larger values of $w$ correspond to the terrace widths below which the energies of the higher harmonics of the wave function are pushed beyond the Fermi level.

also to other (111) surfaces where surface states of the same nature exist. For $\operatorname{Ag}(111)$ and $\operatorname{Pt}(111)$, e.g., we calculate critical TWs of 36.6 and $8.75 \AA$, respectively. We note that a recent STM study by Petersen et al. [25] has shown a bulk contribution to the charge density oscillations near a step with a slightly larger wave vector $\left(0.24\right.$ vs $\left.0.21 \AA^{-1}\right)$. The oscillations arise from a surface resonance corresponding to the neck states on the Fermi surface. Since the critical wave vectors and the dispersion for the resonance and the surface state are similar [16], the critical TW for bulk and surface states should be about the same. We can, therefore, not exclude a contribution of the resonance. Our results, nevertheless, demonstrate clearly that the magnitude of the ES barrier is correlated with the occupation of electronic states localized in the outermost regions of the surface.

It is not understood at present how the occupation of surface states may affect the ES barrier. Memmel and Bertel [15] proposed that the occupation of the surface state enlarges the binding energy of adatoms. According to their model the ES barrier should arise from a lower binding energy near a step due to the boundary condition that the wave function be zero at the step. This model seems to entail the consequence that the binding energy of adatoms on very small terraces with no occupation of the surface state at all should be smaller than on large terraces, approximately by the amount of the ESactivation energy. This in turn would enlarge the energy for the formation of an adatom from a kink site on such a terrace which would balance the effect of the reduction of the ES barrier as far as the interlayer mass flow is concerned [Eq. (1)]. Thus the model should not lead to an enhanced mass flow in island decay experiments. An alternative possibility is that the charge density localized above the surface affects the atom positions. Removing this charge by quantum confinement should lead to a contraction of the distance between the 1st and $2 \mathrm{nd} \mathrm{Cu}$ layer. This contraction would concern the atoms farther away from the step edge while the $\mathrm{Cu}$ atoms next to the step would remain in their positions because the surface charge density is low there anyway due to the boundary condition. The overall effect of removing the charge density by confinement would therefore be a relative change in the vertical position of the 1st layer atoms which could affect in turn the magnitude of the ES barrier.

Stimulating discussion with E. Bertel and T. L. Einstein are gratefully acknowledged. The work was partially supported by the Fond der Chemischen Industrie.

*Author to whom correspondence should be addressed. Electronic address: m.giesen@fz-juelich.de

[1] G. Ehrlich and F. G. Hudda, J. Chem. Phys. 44, 1039 (1966).

[2] R. L. Schwoebel and E. J. Shipsey, J. Appl. Phys. 37, 3682 (1966).

[3] R. Kunkel et al., Phys. Rev. Lett. 65, 733 (1990).

[4] H. A. v. d. Vegt et al., Phys. Rev. Lett. 68, 3335 (1992).

[5] J. Vrijmoeth et al., Phys. Rev. Lett. 72, 3843 (1994).

[6] F. Tsui et al., Phys. Rev. Lett. 76, 3164 (1996).

[7] W. Wulfhekel et al., Surf. Sci. 348, 227 (1996).

[8] M. Karimi et al., Phys. Rev. B 52, 5364 (1995).

[9] Y. Li and A. E. DePristo, Surf. Sci. 319, 141 (1994).

[10] O. S. Trushin et al., Phys. Rev. B 56, 12135 (1997).

[11] P. J. Feibelman, Phys. Rev. Lett. 81, 168 (1998).

[12] M. Kalff et al., Phys. Rev. Lett. 81, 1255 (1998).

[13] G. Schulze Icking-Konert et al., Surf. Sci. 398, 37 (1998).

[14] G. Boisvert and L. J. Lewis, Phys. Rev. B 56, 7643 (1997).

[15] N. Memmel and E. Bertel, Phys. Rev. Lett. 75, 485 (1995).

[16] S. D. Kevan, Phys. Rev. Lett. 50, 526 (1983).

[17] J. Li et al., Phys. Rev. Lett. 80, 3332 (1998).

[18] M. Giesen et al., Phys. Rev. Lett. 80, 552 (1998).

[19] G. Schulze Icking-Konert et al., Jül Report No. 3588, 1998.

[20] D. C. Schlößer et al. (to be published).

[21] M. Giesen et al., Surf. Sci. (to be published).

[22] K. R. Roos and M.C. Tringides, Surf. Rev. Lett. 5, 833 (1998).

[23] P. Stoltze, J. Phys. Condens. Matter 6, 9495 (1994).

[24] G. C. Kallinteris et al., Surf. Sci. 369, 185 (1996).

[25] L. Petersen et al., Phys. Rev. B 58, 7361 (1998). 